\title{
LITERATUR
}

\section{Schwierige Nachbarschaft: Die Nachbarschaftspolitiken der Europäischen Union in der wissenschaftlichen Analyse}

\author{
Andreas Marchetti ${ }^{*}$
}

Die europäische Integration hat seit jeher auf die Staaten ihrer Nachbarschaft ausgestrahlt. Während sich die Beziehungen zu den Nachbarstaaten aber lange Zeit formal nicht weiter von den Außenbeziehungen der später in die Union übergehenden Gemeinschaft unterschieden, unternimmt die Europäische Union seit mittlerweile zehn Jahren Schritte zur ausdrücklichen Hervorhebung und besonderen Ausgestaltung dieser Beziehungen, indem sie neben der Schaffung der Europäischen Nachbarschaftspolitik (ENP) auch eine explizite Berücksichtigung der Nachbarstaaten im europäischen Primärrecht vornahm. ${ }^{1}$ Insbesondere die ENP hat seither zahlreiche Anpassungen und Änderungen erfahren, wobei hier nicht einmal ansatzweise auf ihren vielfältigen Wandel in Reichweite, Anspruch und Design eingegangen werden kann. Zu konstatieren ist aber, dass sich hieraus wie auch aus dem Zusammenspiel beziehungsweise Nebeneinander von Politiken der Europäischen Union und ihrer Mitgliedstaaten eine hoch komplexe Struktur aus Politikverflechtung und -überlagerung im europäischen Nachbarschaftsraum ergeben hat. ${ }^{2}$

\section{Pluralistische Nachbarschaft}

Die hier besprochenen Bände tragen dieser Komplexität Rechnung, indem sie nicht nur auf den an die ENP gekoppelten Nachbarschafts-
Tanja A. Börzel/Katrin Böttger (Hrsg.): Policy Change in the EU's Immediate Neighbourhood: A Sectoral Approach, Europäische Schriften, Band 93, Nomos Verlag: Baden-Baden 2012, ISBN 978-3-8329-7441-1; 174 Seiten, 34,00 €.

Sandra Lavenex/Frank Schimmelfennig (Hrsg.): Democracy Promotion in the EU's Neighbourhood. From Leverage to Governance?, Routledge, London/New York 2013; ISBN 978-0-415-52311-0; 186 Seiten, $\$ 125,00$.

Edmund Ratka/Olga A. Spaiser (Hrsg.): Understanding European Neighbourhood Policies. Concepts, Actors, Perceptions, Münchner Beiträge zur europäischen Einigung, Band 22, Nomos Verlag: Baden-Baden 2012, ISBN 978-3-8329-7203-5; 373 Seiten, 59,00€.

begriff abzielen, sondern ein weiter gefasstes Verständnis von Nachbarschaft zugrundelegen. Nach diesem Grundverständnis lassen sich konsequenter als die ENP dies leistet, diejenigen regionalen Nachbarn der Europäischen Union identifizieren, die aus struktureller Sicht auch zusammen zu betrachten sind. Deren gemeinsames Kennzeichen beschränkt sich nicht nur auf ein Angrenzen an die Europäische Union oder die Verortung in ihrem regionalen Umfeld, sondern liegt zudem und zuvörderst im

* Dr. Andreas Marchetti, Inhaber von poliglott; Senior Fellow, Zentrum für Europäische Integrationsforschung (ZEI) der Universität Bonn.

1 Bereits der Vertrag über eine Europäische Verfassung sah mit Artikel I-57 spezielle Bestimmungen für die Nachbarn der Union vor, die sich nunmehr wortgleich in Artikel 8 des Vertrags über die Europäische Union finden.

2 Von, ,vertikaler und horizontaler Fragmentierung“ sprechen entsprechend auch Edmund Ratka/Olga A. Spaiser: Introduction: Understanding European Neighbourhood Policies, in: Edmund Ratka/Olga A. Spaiser (Hrsg.): Understanding European Neighbourhood Policies. Concepts, Actors, Perceptions, Münchner Beiträge zur europäischen Einigung, Band 22, Baden-Baden 2012, S. 15-23, hier S. 19 (eigene Übersetzung). 
Anspruch der Europäischen Union, in diese Staaten im Sinne einer Transformation hineinzuwirken. ${ }^{3}$ Konsequenterweise werden in den Bänden neben den durch die ENP erfassten Staaten entsprechend auch die - potenziellen Beitrittskandidaten des westlichen Balkans und die Türkei berücksichtigt sowie die Beziehungen zu Russland thematisiert. Folgerichtig findet auch der Terminus Europäische Nachbarschaftspolitik im Singular in den drei rezensierten Sammelbänden nur zurückhaltend Anwendung. Vielmehr ist in ihnen entweder allgemeiner von ,Nachbarschaft" oder aber in der Tat von ,Nachbarschaftspolitiken` im Plural die Rede.

Gemäß der Vielschichtigkeit der europäischen Nachbarschaftspolitiken kann es kaum einen zwingenden und damit einheitlichen Zugang zu diesem breiten Sujet europäischer Politik geben. So befassen sich auch die drei Sammelbände mit dem Thema auf unterschiedliche und in ihrem jeweiligen Anspruch berechtigte Weise.

\section{Europäisierung unter den Bedingungen von Komplexität}

Den umfassendsten Ansatz verfolgt der von Edmund Ratka und Olga A. Spaiser edierte Band, der im Vergleich zu den anderen hier besprochenen Veröffentlichungen auch den größten Umfang aufweist. In vier Abschnitten nähern sich die Autoren den europäischen Politikansätzen in der Nachbarschaft. Im ersten Teil beschäftigen sich vier Beiträge mit der Frage, wie der skizzierten Komplexität analytisch begegnet werden kann, um zentrale Me- chanismen der Nachbarschaftspolitiken besser zu durchdringen. Dabei greifen drei Autoren insbesondere das den gesamten Band durchziehende Konzept der Europäisierung auf und liefern aus ihrer jeweiligen Perspektive Beiträge zur aktuellen Forschung. ${ }^{4}$ Aufgrund der Komplexität des Forschungsgegenstands plädieren die Autoren für eine Weiterentwicklung und gegebenenfalls Kombination bestehender Analyseansätze, für die sie auch konkrete Ansatzpunkte benennen, ohne diese aber zu vollständigen Erklärungsmodellen auszuformulieren. Da man ein einziges Erklärungsmodell für die Nachbarschaftspolitiken der Europäischen Union ohnehin nicht erwarten kann, stellen die Beiträge gute und wichtige Impulse für die weitere Auseinandersetzung mit dem Thema dar, indem insbesondere die Notwendigkeit stärkerer Kontextualisierung und Differenzierung in der Forschung zu Europäisierungsprozessen angemahnt wird. ${ }^{5}$ Als zweiter und dritter Teil des Buches folgen Beiträge entlang der landläufigen und auch seitens der Europäischen Union vorgenommenen Unterteilung in die Beziehungen zu Nachbarn im Süden und Nachbarn im Osten. Allerdings will dies hier nicht originell und weiterführend erscheinen, zumal damit die analytische Einheit des ersten Teils wieder aufgehoben wird, ohne dann aber konsequent betrieben zu werden, da nicht alle subregionalen Einheiten in der östlichen und südlichen Nachbarschaft gleichermaßen Berücksichtigung erfahren. Im vierten Teil des Bandes widmen sich mehrere Autoren der Demokratieförderung und damit einem Politikfeld, dem zwar in politischen Zielerklärungen stets besondere Bedeutung sowie in der Wissenschaft großes Interesse entgegengebracht

3 Dies gilt nicht für westeuropäische Staaten, die nicht Mitglied der Europäischen Union sind, weshalb diese auch nicht in die ENP einbezogen sind.

4 Auf den vierten Beitrag dieses ersten Teils, der sich dem Thema aus strategischer Sicht widmet, wird am Schluss dieser Rezension noch gesondert verwiesen.

5 Vgl. Patrick Müller: Europeanisation within and beyond the EU: A Conceptual Overview, in: Ratka/Spaiser (Hrsg.): Understanding European Neighbourhood Policies, 2012, S. 37-51, hier S. 46-48; Christine Normann: Analysing the Evolution of the European Neighbourhood Policy: Theoretical and Methodological Triangulation, in: Ratka/Spaiser (Hrsg.): Understanding European Neighbourhood Policies, 2012, S. 53-68, hier S. 65-66; Bernd Weber: The Evaluation of the EU's Neighbourhood Policies: How to Measure the Effectiveness of External Europeanisation, in: Ratka/Spaiser (Hrsg.): Understanding European Neighbourhood Policies, 2012, S.69-86, hier S. 82-83. 
wird, das allerdings in der politischen Praxis keineswegs als das unangefochtene Hauptanliegen europäischer Nachbarschaftspolitiken gelten kann. Dass sich die Herausgeber und ihre Autoren dessen bewusst sind, steht außer Frage, die deutliche Hervorhebung des Politikfelds Demokratieförderung erscheint mit Blick auf die breite Anlage des Bandes dann aber nur bedingt nachvollziehbar. Weshalb darüber hinaus in einem auf Englisch erschienenen Band, der zudem als deutsch-französische Co-Produktion zwischen dem Münchner Centrum für angewandte Politikforschung und Sciences Po Paris zu sehen ist, relativ häufig ein ausdrücklicher Fokus auf Deutschland gelegt wird, ohne andere Mitgliedstaaten oder zumindest aber Frankreich einer ähnlich vertieften Betrachtung zu unterziehen, bleibt offen. Hier hätte sich angesichts der gegebenen faktischen Politikfragmentierung und der breiten Anlage des Bandes ein weiterer eigenständiger Teil zu mitgliedstaatlichen Perzeptionen, Präferenzen und Politiken angeboten.

\section{Europas Transformationskraft auf dem Prüfstand}

Einer Auswahl von Politikbereichen, die in die Nachbarschaft gerichtet sind, widmet sich der von Tanja A. Börzel und Katrin Böttger verantwortete Band, der sich seinerseits als Gemeinschaftswerk des Berliner Instituts für Europäische Politik sowie der Freien Universität Berlin präsentiert. Dieser Band stellt die Transformationskraft und besonders -fähigkeit der Europäischen Union in ihrer Nachbarschaft in den Mittelpunkt der Erwägungen ${ }^{6}$ und beschäftigt sich damit ebenso wie der bereits besprochene Band auch mit dem Phänomen externer Europäisierung. ${ }^{7}$ Dabei wird ohne Anspruch auf Vollständigkeit ein breiter thematischer Bogen gespannt. Dieser umfasst die Beitrittsperspektiven Serbiens und des Kosovo ebenso wie die Frage des Minderheitenschutzes in der Türkei. Er reicht vom Verhältnis der Europäischen Union zur Zivilgesellschaft in osteuropäischen ENP-Staaten über die Möglichkeiten zunehmender Konvergenz im Regulierungsbereich in der Ukraine bis hin zur Visaliberalisierung mit dem Südkaukasus. Ebenfalls wird die Förderung marokkanischer Umweltpolitik thematisiert wie auch die Demokratieförderung mit Blick auf den südlichen Mittelmeerraum, hier aber als eines unter mehreren Politikfeldern. Der geografische Fokus der Einzelbeiträge ist unterschiedlicher Natur, da die jeweiligen Bereiche entweder entlang der Klassifizierung östliche Nachbarschaft südliche Nachbarschaft oder aber am Beispiel einzelner Nachbarstaaten untersucht werden. Durch das offensichtlich exemplarische Vorgehen stellt sich im Falle dieses Bandes der Eindruck des Zufälligen nicht ein, zumal es den Herausgeberinnen gelungen ist, die Autorinnen auf eine weitgehend einheitliche Grundstruktur ihrer Beiträge zu verpflichten: Ausgehend von einer Analyse der Problem- beziehungsweise Ausgangslage stellen die Autorinnen Überlegungen darüber an, wie die Europäische Union ihre Politik im jeweiligen Fall zur besseren Zielerreichung umgestalten müsse und wie dies konkret in der Politikumsetzung zu leisten sei. Auch wenn gerade dieser letzte Teil doch meist sehr knapp ausfällt, ist der Band klar policy-orientiert, dabei aber informiert und wissensbasiert, sodass es die Autorinnen zu vermeiden wissen, in politisches Wunschdenken abzugleiten, sondern in der Tat nachdenkenswerte Vorschläge zur Politikfortentwicklung unterbreiten. Hervorzuheben sind hier unter anderem die Einforderung besserer politischer Kommunikation sowie das plausible Plädoyer für einen stärkeren Einbezug zivilgesellschaftlicher aber auch privatwirtschaftlicher Akteure zur Erreichung erklärter

6 Katrin Böttger/Tanja A. Börzel: Preface in: Tanja A. Börzel/Katrin Böttger (Hrsg.): Policy Change in the EU's Immediate Neighbourhood: A Sectoral Approach, Europäische Schriften, Band 93, Baden-Baden 2012, S. 9-12, hier S. 9; Tanja A. Börzel/Katrin Böttger: Conclusion: The Power to Transform Lies in the Detail, in: Börzel/ Böttger (Hrsg.): Policy Change in the EU's Immediate Neighbourhood, 2012, S. 163-171, hier S. 163.

7 Börzel/Böttger: Conclusion, 2012, S. 167-169. 
Politikziele. ${ }^{8}$ Besonders brauchbar für eine schnelle Orientierung im Band erweisen sich schließlich die den Einzelbeiträgen vorangestellten ,executive summaries', wie sie sich üblicherweise nur bei Zeitschriftenaufsätzen finden.

\section{Schwierigkeiten der Demokratieförderung}

Die fokussierteste und systematischste Sammlung von Beiträgen legen Sandra Lavenex und Frank Schimmelfennig mit ihrem Band vor, dessen Beiträge zuvor bereits in einer Sondernummer der Zeitschrift Democratization publiziert wurden. ${ }^{9}$ Die Autoren beschäftigen sich ausschließlich mit der Frage nach den Möglichkeiten und Grenzen der Demokratieförderung der Europäischen Union in ihrer Nachbarschaft. Hierzu stellen die beiden Herausgeber zunächst drei idealtypische Modelle der Demokratieförderung vor: ${ }^{10}$ Während das Linkeage-Modell auf die Förderung demokratischer Kultur in der Gesellschaft durch transnationale Vernetzung mithilfe von Sozialisierungsprozessen von unten nach oben zielt, fokussiert im Gegensatz hierzu das LeverageModell durch die Nutzung intergouvernementaler Kanäle auf die Schaffung demokratischer Institutionen vermittels Konditionalität und setzt damit auf einen Prozess von oben nach unten. Als Zwischenmodell kann das besonders seitens der Herausgeber als Alternative angebotene Governance-Modell verstanden werden, das sektoriell ansetzt und über transgouvernementale Verknüpfungen wiederum durch Sozialisierung zu demokratischer
Governance beitragen soll. Ausgehend von dieser Diskussion konzentrieren sich die Beiträge des Bandes auf das Leverage- und das Governance-Modell, während das LinkeageModell aufgrund seines vergleichsweise schwachen Einflusses auf konkrete Demokratisierungsprozesse nur bedingt berücksichtigt wird. Die ersten drei Beiträge des Bandes zeigen die spezifischen Grenzen europäischer Demokratieförderung auf Basis des LeverageModells in der Türkei, in Tunesien und Marokko sowie in der Ukraine auf, wobei Paul Kubicek in seiner Untersuchung zur Türkei auch systematisch auf das Linkeage-Modell eingeht. ${ }^{11}$ Dem folgen weitere drei Beiträge, die sich nunmehr den Möglichkeiten der Demokratieförderung auf Basis des GovernanceModells zuwenden. Darunter fällt zunächst der sehr aufschlussreiche Artikel von Anne Wet$z e l$, in dem sie die Grenzen des GovernanceModells gerade im Falle betonter wirtschaftlicher Interessen der Europäischen Union aufzeigt. ${ }^{12}$ Besonders lesenswert ist auch der abschließende Beitrag zur Demokratieförderung im Rahmen der ENP, in dem vergleichend in drei Sektoren Moldawien und die Ukraine sowie Jordanien und Marokko untersucht werden. Durch die hier erfolgte und viel zu selten vorgenommene Durchbrechung des weit verbreiteten separaten Blicks auf die Ost- und Südnachbarschaft der Europäischen Union wird das bemerkenswerte Ergebnis zutage gefördert, dass sich bei allen notwendigen Einschränkungen zur Verallgemeinerung die strukturellen Charakteristika der Adressatenstaaten als weniger bedeutsam erweisen als an-

8 Mariella Falkenhain/Iryna Solonenko: The EU and Civil Society in Eastern Europe: Partners in the Pursuit of Policy Change?, in: Börzel/Böttger (Hrsg.): Policy Change in the EU's Immediate Neighbourhood, 2012, S. 56-76, hier S. 72-73; Julia Langbein: Achieving Regulatory Convergence in the European Neighbourhood: What's the Right Toolbox?, in: Börzel/Böttger (Hrsg.): Policy Change in the EU's Immediate Neighbourhood, 2012, S. 77-97, hier S. 94.

9 Democratization 4/2011: Democracy promotion in the EU's neighbourhood: from leverage to governance?

10 Im folgenden Sandra Lavenex/Frank Schimmelfennig: EU democracy promotion in the neighbourhood: from leverage to governance?, in: Sandra Lavenex/Frank Schimmelfennig (Hrsg.): Democracy Promotion in the EU's Neighbourhood. From Leverage to Governance?, London/New York 2013, S. 1-25, hier S. 5-14.

11 Paul Kubicek: Political conditionality and European Union's cultivation of democracy in Turkey, in: Lavenex/ Schimmelfennig (Hrsg.): Democracy Promotion in the EU's Neighbourhood, 2013, S. 26-47.

12 Anne Wetzel: The promotion of participatory governance in the EU's external policies: compromised by sectoral economic interests?, in: Lavenex/Schimmelfennig (Hrsg.): Democracy Promotion in the EU's Neighbourhood, 2013, S. 94-116, hier S. 106. 
zunehmen wäre; demgegenüber weisen die untersuchten Staaten aber gleichermaßen eine klare Diskrepanz zwischen formaler Regelanpassung und konkreter Regelumsetzung auf, ${ }^{13}$ sodass sich für die Politiken der Europäischen Union weniger die Frage nach dem Wesen des jeweiligen Nachbarstaates stellt als vielmehr die Frage nach dem richtigen Ansatzpunkt.

\section{Die EU als semi-strategischer Akteur in ihrer Nachbarschaft?}

In der Summe liefern die drei besprochenen Sammelbände gute Impulse und wichtige Ansätze zu einem vertieften Verständnis der europäischen Nachbarschaftsbeziehungen. Davon ausgehend präsentieren sich als lohnenswerte Pfade für weiterführende Untersuchungen zum einen der konzentrierte Fokus auf spezifische Sektoren, zum anderen die Wahl komparativer, transregionaler Ansätze, um jenseits des Ost-Süd-Blicks auf die Nachbarschaft vermehrt Erkenntnisse struktureller Natur in Bezug auf die eingesetzten Politikinstrumente zu gewinnen. Diese könnten dann auch zur Stärkung der Effektivität und Effizienz europäischer Politik nutzbar gemacht werden, ein Anliegen, das neben dem wissenschaftlichen Erkenntnisinteresse den drei Sammelbänden keineswegs fern ist und zu dem sie ihren jeweils eigenen Beitrag leisten. Immerhin ist den meisten Beiträgen die Frage gemein, wie die Europäische Union ihr Handeln in ihrer Nachbarschaft verbessern kann, sodass konsequenter als bisher Politikergebnisse erreicht werden können, die auch den formulierten Politikzielen entsprechen.
Schlussendlich bleibt aber weiterhin die übergeordnete Frage nach dem strategischen Ansatz der Europäischen Union in ihrer Nachbarschaft und damit ihrer Akteursqualität bestehen. Obwohl bereits früh mit Blick auf den ersten konzeptionellen Entwurf zu einer ausdrücklichen Nachbarschaftspolitik der Europäischen Union geurteilt wurde, dass es sich dabei um ein ,erste[s] geostrategische[s] Dokument ${ }^{\star 14}$ der Europäischen Union handele, lautet doch seither die durchgängige und auch in den hier besprochenen Bänden verbreitete Einschätzung, dass die Europäische Union nicht wirklich als strategischer Akteur in ihrer Nachbarschaft begriffen werden könne, weise sie doch deutliche strategische Defizite auf. ${ }^{15}$ Diese Einschätzung geht fast immer einher mit der normativen Forderung, die Europäische Union solle diesen gegenwärtigen Zustand baldmöglichst überwinden. ${ }^{16}$ Gleichwohl bleibt offen, als was die Europäische Union aus strategischer Sicht gegenwärtig zu begreifen ist. Immerhin befindet sie sich - mit Blick auf die Dauerhaftigkeit, mit der ihr schon seit Jahren abgesprochen wird, uneingeschränkt strategischer Akteur zu sein - womöglich gar nicht in einem dynamischen Prozess der strategischen Ausrichtung, sondern bleibt zumindest mittelfristig in dem - normativ unbefriedigenden - heutigen Zustand. So wie sich die Europäische Union als Gebilde sui generis präsentiert, liegt die Vermutung nahe, dass sie auch im besten Falle strategischer Akteur sui generis ist, der spezifische Eigenheiten aufweist, die in ihrer Natur und den ihr zugrundeliegenden Mechanismen begründet liegen. Selbstverständlich bedarf diese Einschätzung, um analytisch

13 Tina Freyburg/Sandra Lavenex/Frank Schimmelfennig/Tatiana Skripka/Anne Wetzel: Democracy promotion through cooperation? The case of the European Neighbourhood Policy, in: Lavenex/Schimmelfennig (Hrsg.): Democracy Promotion in the EU's Neighbourhood, 2013, S. 142-170, hier S. 161.

14 Ulrike Guérot/Andrea Witt: Europas neue Geostrategie, in: Aus Politik und Zeitgeschichte 17/2004, S. 6-12, hier S. 11.

15 Vgl. Werner Weidenfeld: Europe's International Responsibility: A Continent in Search of its Strategy, in: Ratka/ Spaiser (Hrsg.): Understanding European Neighbourhood Policies, 2012, S. 25-33; Ludwig Schulz: A Strategy Theory Approach to the Analysis of European Foreign Policy, in: Ratka/Spaiser (Hrsg.): Understanding European Neighbourhood Policies, 2012, S. 87-105, hier S. 98-102.

16 So auch Schulz: A Strategy Theory Approach, 2012, S. 103, der aber zugleich systematisch das strategische Defizit der Europäischen Union in Augenschein nimmt. 
sinnvoll und politisch hilfreich sein zu können, weiterer Konkretisierung. Dabei deutet vieles darauf hin, dass die Europäische Union tatsächlich als ein semi-strategischer Akteur zu verstehen ist. ${ }^{17}$ Als solcher weist sie zwar a) über die Zielformulierung und die grundsätzliche Bereitschaft des Mitteleinsatzes zur Zielerreichung durchaus Züge eines strategischen Akteurs auf, sie unternimmt aber b) keine konsequente Priorisierung von Zielen, verbunden mit einem weitgehend reaktiven Akteursverhalten, sodass eine hohe Anfälligkeit für Inkonsequenz und schwaches Durchhaltevermö- gen besteht. ${ }^{18}$ Auf diesen Erwägungen aufbauend könnten klarere Aussagen über das Wesen der Europäischen Union aus strategischer Sicht getroffen werden, um auch die den Nachbarschaftspolitiken - nach innen wie außen - zugrundeliegenden Mechanismen noch besser zu verstehen, um dann wiederum in normativer Absicht Möglichkeiten der effektiveren Politikformulierung und -umsetzung zu entwickeln. Immerhin ist der Nachbarschaftsraum der Europäischen Union zu bedeutend als dass sie sich hier ein Scheitern ihrer Politiken erlauben könnte.

17 Adrian Hyde-Price: A neurotic 'centaur': the limitations of the EU as a strategic actor, in: Kjell Engelbrekt/Jan Hallenberg (Hrsg.): The European Union and Strategy. An emerging actor, London/New York 2008, S. 153-166, hier S. 165, bezeichnet die Europäische Union in ähnlicher Weise als „,schwachen strategischen Akteur“ (eigene Übersetzung).

18 Dieser Versuch einer ersten Abgrenzung in Ableitung von den bei Schulz: A Strategy Theory Approach, 2012, S. 96-97 an einen strategischen Akteur angelegten Kriterien. 